\title{
The association of physical activity with obesity, fat distribution and glucose intolerance in Pima Indians
}

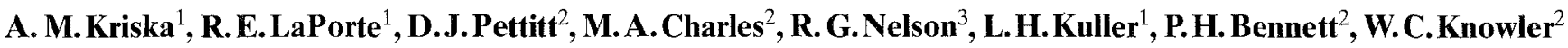 \\ ${ }^{1}$ Department of Epidemiology, University of Pittsburgh, Pittsburgh, Pennsylvania, USA \\ ${ }^{2}$ Diabetes and Arthritis Epidemiology Section, National Institute of Diabetes, Digestive and Kidney Diseases, Phoenix, Arizona, USA \\ ${ }^{3}$ Department of Biostatistics and Epidemiology, The Cleveland Clinic Foundation, Phoenix, Arizona, USA
}

\begin{abstract}
Summary. The relationships between physical activity, obesity, fat distribution and glucose tolerance were examined in the Pima Indians who have the highest documented incidence of non-insulin-dependent diabetes. Fasting and 2-h post-load plasma glucose concentrations, body mass index, and waist-to-thigh circumference ratios were determined in 1054 subjects aged 15-59 years. Current (during the most recent calendar year) and historical (over a lifetime) leisure and occupational physical activity were determined by questionnaire. Current physical activity was inversely correlated with fasting and 2 -h plasma glucose concentrations, body mass index and waist-to-thigh ratios for most sex-age groups even when diabetic subjects were excluded. Controlled for age, obesity and fat distribution, activity remained significantly associated with 2 -h plasma glucose concentrations in males. In subjects aged 37-59 years, individuals with diabetes compared to those without reported significantly less leisure
\end{abstract}

physical activity during the teenage years (median hours per week of activity, 9.1 vs 13.2 for men; 1.0 vs 2.2 for women). Controlled for body mass index, sex, age and waist-to-thigh ratio, subjects who reported low levels of historical leisure physical activity had a higher rate of diabetes than those who were more active. In conclusion, current physical activity was inversely related to glucose intolerance, obesity and central distribution of fat, particularly in males. Subjects with diabetes were currently less active and reported less historical physical activity than non-diabetic subjects. These findings suggest that activity may protect against the development of non-insulin-dependent diabetes both directly and through an influence on obesity and fat distribution.

Key words: Physical activity, obesity, fat distribution, glucose intolerance, Pima Indians, exercise.
The relationship between physical activity and non-insulin-dependent diabetes mellitus is important since physical activity is a potentially modifiable behaviour and non-insulin-dependent diabetes is one of the leading causes of death and disability in the United States [1]. Clinical studies have identified mechanisms through which physical activity might influence the development of non-insulin-dependent diabetes, such as by increasing insulin sensitivity [2] or preventing obesity or beneficially altering fat distribution [3] or both. Support for these clinical findings has been extended recently to selected populations (i.e. college graduates and nurses) $[4,5]$ in which the relationship of a physically active lifestyle to the development of non-insulin-dependent diabetes was examined. However, since the diagnosis of diabetes in these studies was based upon self-report and since national data have demonstrated that almost half of the cases of non-insulin-dependent diabetes are undetected [1], the extent and strength of the relationship between physical activity and non-insulin-dependent diabetes need to be examined in a population-based study that determines diabetes based upon oral glucose tolerance tests. A population at high risk for development of noninsulin-dependent diabetes would be ideally suited for such an investigation.

The relationship between physical activity and noninsulin-dependent diabetes was, therefore, investigated in a population of Native Americans, the Pima Indians, who have the highest documented incidence rates of noninsulin-dependent diabetes in the world $[6,7]$. Physical activity was assessed in this population by means of a carefully validated activity questionnaire [8]. The purpose of this current effort was to examine the initial 2 years of questionnaire data in order to provide information about the cross-sectional association between physical activity and glucose intolerance and insight into possible mediators of this association such as obesity or fat distribution. 
Table 1. General characteristics of 1054 Pima Indians

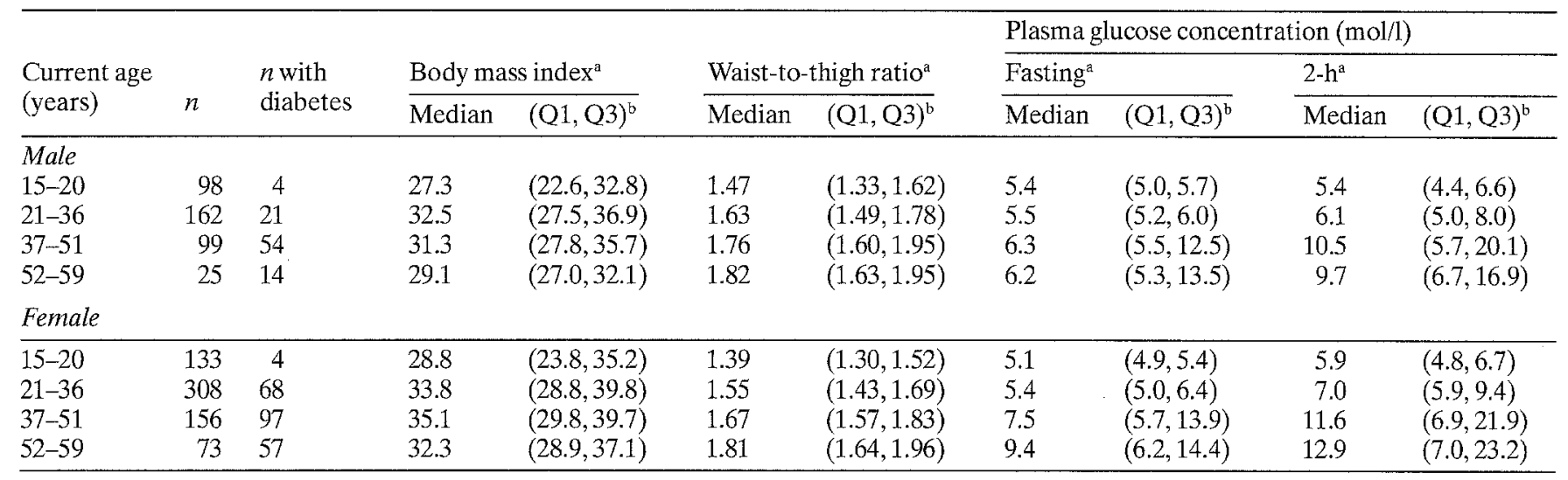

${ }^{a}$ Number of missing values for each variable are 1 for body mass index, 220 for waist-to-thigh ratio, 99 for fasting, and 41 for 2 -h plasma glucose concentration; ${ }^{b}$ numbers in parentheses represent the 25 th and 75 th percentiles

\section{Subjects, materials and methods}

\section{Study description}

Pima Indians of the Gila River Indian Community of Arizona have been the subjects of a longitudinal population-based diabetes research study conducted by the National Institute of Diabetes and Digestive and Kidney Diseases since 1965. Approximately 5000 Pina Indians over the age of 5 years currently live in or near the designated study area and constitute the study population. At intervals of approximately 2 years, each subject is invited for a comprehensive examination conducted at the study clinic located in the community $[6,7]$.

At each examination, an oral glucose tolerance test (OGTT) is performed, in which venous plasma glucose concentrations are determined after an overnight fast and $2 \mathrm{~h}$ after the ingestion of a 75 $\mathrm{g}$ carbohydrate load. Diabetes is diagnosed if the 2 -h post-load plasma glucose concentration is at least $11.1 \mathrm{mmol} / 1$ [9] at this examination or during the course of routine medical care [7]. The examination also includes a medical history, physical examination, and measurement of height and weight (with light indoor clothing but without shoes). Obesity is estimated by the body mass index or BMI $\left(\mathrm{kg} / \mathrm{m}^{2}\right)$. Body fat distribution is estimated by the ratio of waist circumference (at the level of the umbilicus with the subject supine) to thigh circumference (measured at the highest level of the right thigh with the subject standing). Also at this examination, a physical activity questionnaire is administered by trained interviewers while the OGTT is performed.

\section{Physical activity interview}

Since September 1987, a physical activity questionnaire has been administered to individuals between the ages of 15 and 59 years who attend the study clinic. This questionnaire was shown to be both feasible and reliable in the Pima Indian population. The activity questionnaire, interviewer's instructions and questionnaire calculations have been described previously [8].

The physical activity questionnaire assesses physical activity both currently (leisure and occupational activity over the most recent year and week) and over a lifetime (historical leisure physical activity). Only physical activities that demand energy expenditure greater than that required by activities of daily living such as bathing, grooming and feeding are assessed. An estimate of the individual's physical activity level is determined for each period of his/her life and expressed as hours per week, or alternatively can be weighted by a crude estimate of the metabolic cost of the activity (known in the exercise physiology literature as METs) and expressed as METhours per week. One MET represents the energy expenditure for an individual at rest, whereas a $10 \mathrm{MET}$ activity requires 10 times the resting energy expenditure [10].

To determine the relationship between physical activity and glucose tolerance, obesity, or fat distribution, all physical activities reported in the questionnaire were included in the activity estimate. In addition, to examine the specific contribution of vigorous activities, (i. e. those activities estimated to be strenuous enough to elicit a cardiovascular training effect resulting in improvement of physical fitness), only activities of 6 METs or more were summed [8]. Pregnant women were excluded from all analyses as pregnancy may influence their current physical activity level, preventing a valid measurement of their usual activity.

A subjective determination was made by the interviewer whether or not the participant was capable of correctly answering the activity questions during the activity interview. Interviews judged "not reliable" by the trained interviewer were eliminated from the analyses [8].

As the reporting of walking for exercise had previously been shown to be unreliable $[8,11]$, particularly for determination of historical or past year (compared with past week) physical activity levels, all summary physical activity estimates in this paper were calculated from data that excluded walking for exercise. Age groups reported in these analyses are based upon the structure of the physical activity questionnaire [8].

\section{Statistical analysis}

Prevalence rates were standardized by the direct method of age adjustment using the Census of the United States Caucasian population, including armed forces, 1980 [7, 12]. Spearman rank-order correlation coefficients were used to assess the bivariate associations between physical activity estimates and fasting glucose, post-load glucose, BMI and waist-to-thigh ratio in each age group. Multiple regression analyses were performed to examine the independent association of current physical activity to fasting and post-load plasma glucose concentrations. In these analyses, the logarithm of glucose concentration and BMI, and the square root of current (past year) physical activity were used to improve the fit of the models, according to analysis of the residuals.

In subjects aged 37 to 59 years, the association of historical leisure physical activity (dichotomized at the median value into high and low physical activity) and the presence of diabetes was also determined. The younger age groups were not included in this analysis because of their lower prevalence of diabetes and the brief time period available for estimating historical physical activity. Multiple 


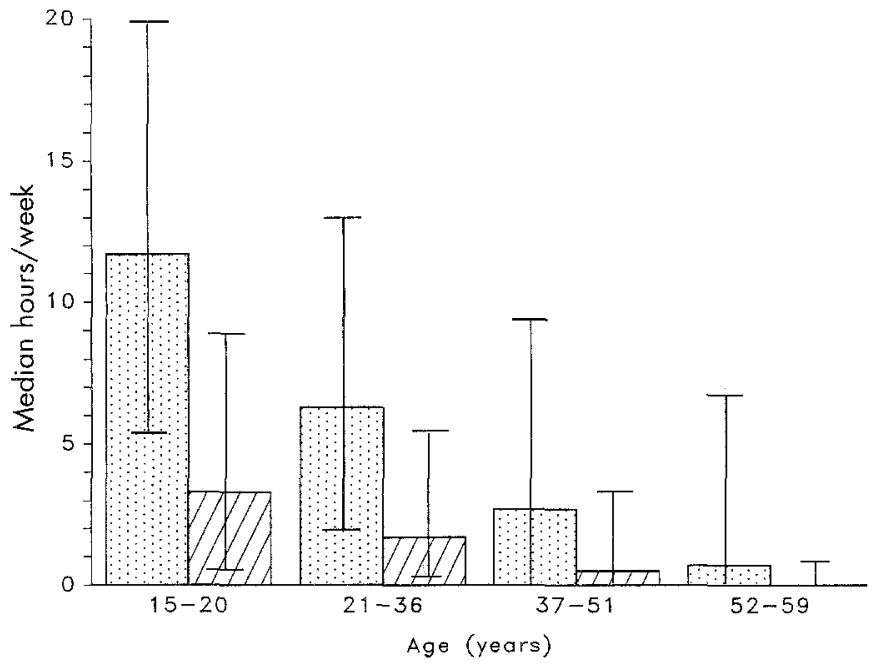

Fig. 1. Past year leisure physical activity in 1054 Pima Indians, (: $:$ male, $\square$ female). Median hours per week of leisure physical activity, by sex, averaged over the past year. The leisure physical activity estimate was calculated excluding walking for exercise. The 25 th and 75 th percentiles are represented by the high and low points of the error bars

logistic regression was used to examine the independent association of historical leisure physical activity, controlled for age, sex, waist-tothigh ratio and $\mathrm{BMI}$, with the presence of non-insulin-dependent diabetes.

\section{Results}

From the time the activity questionnaire was incorporated into the comprehensive examination in September 1987 until September 1989, 'reliable' physical activity interviews were completed on 1054 males and non-pregnant females between the ages of 15 and 59 years whose heritage was at least half Pima, Tohono-O'odham, or a combination of these two closely-related tribes. This represented $78 \%$ of the population who were examined at the clinic during the same time period and reflected a similar proportion of males and females. Nineteen interviews $(1.8 \%)$ were excluded because they were judged 'not reliable' by the interviewer. Limited availability of trained interviewers was the major reason for not obtaining physical activity information from the remainder of the population.

Baseline characteristics of these 1054 individuals are presented in Table 1. BMI and waist-to-thigh ratios were high compared with most other populations, in agreement with previous studies $[13,14]$. Of the total group, 319 $(30 \%)$ had diabetes, a prevalence similar to that in individuals who were examined in the clinic but were not given the physical activity interview.

\section{Current physical activity and non-insulin-dependent diabetes}

Reported leisure physical activity levels during the past year, expressed in hours per week of activity, are presented in Figure 1. Median values are given because the physical activity distributions for most of the age-sex groups are positively skewed. Since the only age-sex group with a median occupational activity value above zero was the 21-36 year old males, only leisure physical activity is presented. This indicated that more than half of the subjects in the other age-sex groups were sedentary in their occupations.

Leisure physical activity was inversely associated with age in both sexes. In each age group, males were more active than females. Past week physical activity estimates (data not shown) exhibited the same general trends. Although all summary physical activity estimates in this paper are calculated from data that excluded walking for exercise, results from all analyses below are similar if walking for exercise is included.

Figure 2 shows the age-adjusted prevalence of non-insulin-dependent diabetes by tertile of past year leisure physical activity in males and females in two age groups (15-36 and 37-59 years). In general, the prevalence of diabetes was inversely related to the level of past year leisure physical activity, although this association was statistically significant $(p<0.001$; Mantel Haenzel chi-square
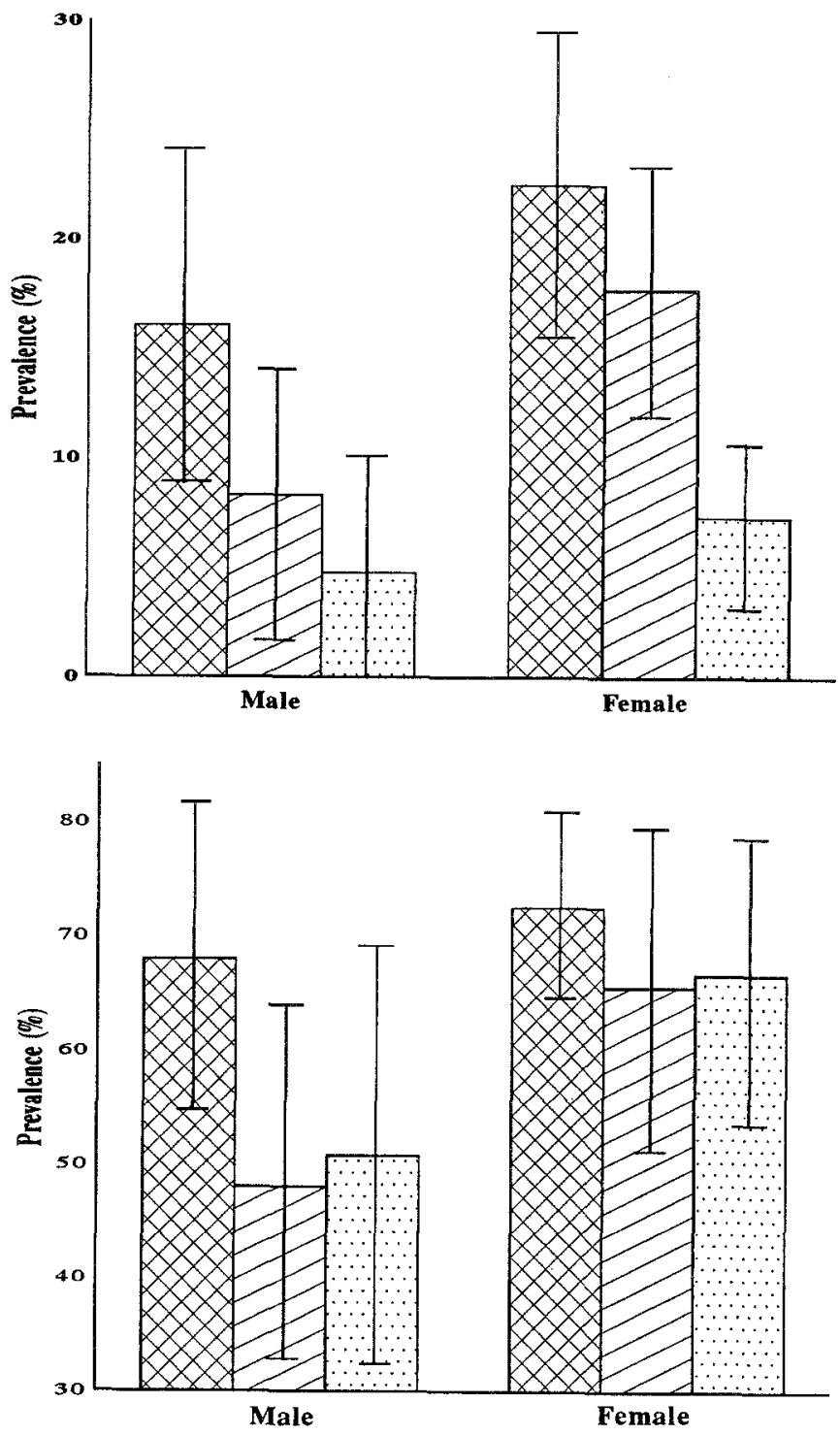

Fig.2. Age-adjusted prevalence of non-insulin-dependent diabetes by tertile of past year leisure physical activity in subjects aged 15-36 (upper panel) and 37-59 years (lower panel). Physical activity level: low, $\nabla$ middle, $::$ high. The error bars represent the $95 \%$ confidence intervals 
Table 2. Spearman correlation coefficients between reported leisure and occupational physical activity (MET-hours per week) and fasting and 2-h plasma glucose concentration, body mass index and waist-to-thigh ratio in male Pima Indians ${ }^{\mathrm{a}}$

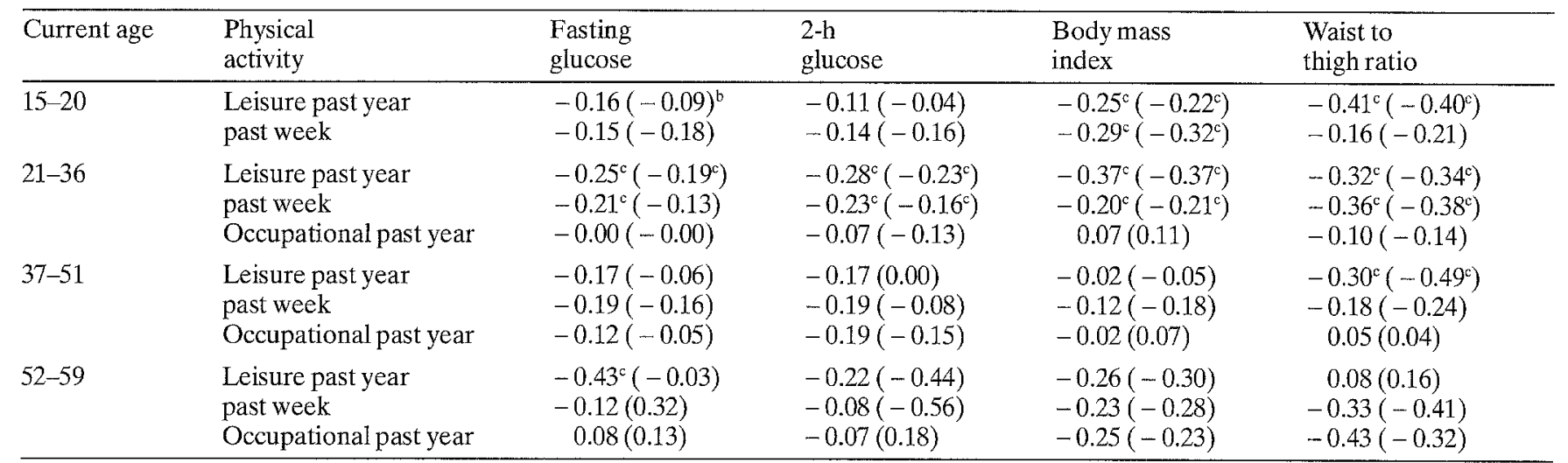

${ }^{a}$ Sample sizes are given in Table $1 .{ }^{b}$ Numbers in parentheses represent correlation coefficients omitting non-insulin-dependent diabetic subjects. (In the oldest age group, sample size was less than 10 ); ${ }^{c} p<0.05$

Table 3. Spearman correlation coefficients between reported leisure and occupational physical activity (MET-hours per week) and fasting and 2-h plasma glucose concentration, body mass index and waist-to-thigh ratio in non-pregnant female Pima Indians ${ }^{\mathrm{a}}$

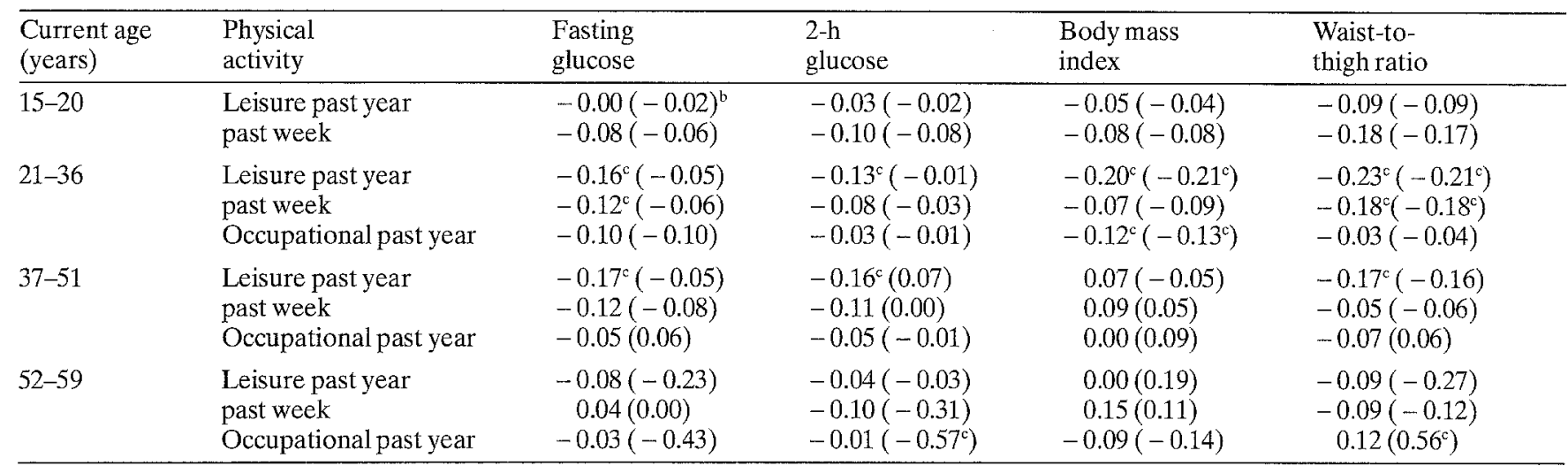

${ }^{a}$ Sample sizes are given in Table 1. ${ }^{b}$ Numbers in parentheses represent correlation coefficients omitting non-insulin-dependent diabetic subjects. (In the oldest age group, sample size was less than 15); ${ }^{c} p<0.05$

analysis controlled for age and sex) only in the younger age group.

\section{Current physical activity and glucose tolerance}

Correlations between current leisure and occupational physical activity with fasting and 2-h plasma glucose concentrations, BMI, and waist-to-thigh ratio are presented for each age group in Tables 2 (males) and 3 (females). Negative relationships between leisure physical activity and these variables were generally found. However, these relationships were not as strong for occupational activity as for leisure activity. The low levels of occupational activity reported may account for these findings.

When individuals with non-insulin-dependent diabetes were excluded from the analysis, significant bivariate relationships between physical activity and glucose concentrations, BMI and waist-to-thigh ratio were still found in the younger but not the older age groups. The lack of significance of the relationships in many of the older age groups may partially be due to the small numbers of individuals remaining once those with non-insulin-dependent diabetes were eliminated. If each physical activity estimate was expressed in hours perweek instead of MET-hours per week, the correlations were generally weaker. In contrast, correlations were considerably stronger in most sex-age groups if the composite physical activity estimate included only those activities that demanded a relatively higher metabolic level, i. e. $6 \mathrm{METs}$ or more (data not shown).

Current physical activity (combined estimate of leisure and occupational past year physical activity) was inversely correlated with both BMI (rho $=-0.20, p<0.001$ ) and waist-to-thigh ratio (rho $=-0.19, p<0.001$ ) in the total sample. Similar relationships were identified when the data were stratified by sex and age groups (with leisure and occupational physical activity shown separately) as noted in Tables 2 and 3. In multiple regression analysis, current physical activity, controlled for age and sex, was significantly associated with both waist-to-thigh ratio and BMI (not shown).

Finally, in all non-diabetic individuals, multiple regression analysis was used to determine if physical activity was independently associated with both fasting and 2-h plasma glucose concentrations. In males but not females, current physical activity (leisure and occupational), control- 
Table 4. Independent ${ }^{\mathrm{a}}$ association of 2-h plasma glucose concentrations with reported past year leisure and occupational physical activity in 15-59-year-old non-diabetic Pima Indians: multiple regression analysis

\begin{tabular}{|c|c|c|c|}
\hline \multirow[b]{2}{*}{ Variable } & \multicolumn{3}{|c|}{$\begin{array}{l}\text { Dependent variable }=2 \text {-h post }- \\
\text { load glucose } \mathrm{b}^{\mathrm{b}}\end{array}$} \\
\hline & $\begin{array}{l}\text { Non-stan- } \\
\text { dardized } \\
\text { regression- } \\
\text { coefficient }\end{array}$ & $\begin{array}{l}\text { Standard } \\
\text { error }\end{array}$ & $p$ value \\
\hline \multicolumn{4}{|l|}{ Males $(n=291)$} \\
\hline Age (years) & 0.002 & 0.002 & 0.427 \\
\hline Body mass index $\left(\mathrm{kg} / \mathrm{m}^{2}\right)^{\mathrm{b}}$ & 0.303 & 0.080 & 0.001 \\
\hline Waist-to-thigh ratio & 0.128 & 0.084 & 0.130 \\
\hline Past year activity (MET-hours) ${ }^{c}$ & -0.010 & 0.003 & 0.005 \\
\hline \multicolumn{4}{|l|}{ Females $(n=444)$} \\
\hline Age (years) & 0.004 & 0.001 & 0.004 \\
\hline Body mass index $\left(\mathrm{kg} / \mathrm{m}^{2}\right)^{\mathrm{b}}$ & 0.407 & 0.059 & 0.001 \\
\hline Waist-to-thigh ratio & -0.152 & 0.084 & 0.071 \\
\hline Past year activity (MET-hours) ${ }^{c}$ & 0.001 & 0.003 & 0.657 \\
\hline
\end{tabular}

all two-way interaction terms involving physical activity did not significantly add to the model, so they were not included. ${ }^{5}$ The logarithm (base 10) of the variables was used in the model. ${ }^{c}$ The square root of physical activity was used in the model. MET, Metabolic cost of activity

Table 5. The association between historical and past year leisure physical activity and prevalence of non-insulin-dependent diabetes in 353 Pima Indians aged 37-59 years: logistic regression

\begin{tabular}{llll}
\hline Time period & & $\begin{array}{l}\text { Odds ratio } \\
(95 \% \text { confi- } \\
\text { dence interval) }\end{array}$ & . \\
\hline Historical & 12-18 years old & $0.45(0.27,0.75)$ & 0.002 \\
activity estimate & $19-34$ years old & $0.47(0.28,0.78)$ & 0.004 \\
Current activity & Past year & $0.68(0.41,1.13)$ & 0.133 \\
estimate & (MET-hours) & & \\
\hline
\end{tabular}

${ }^{a}$ Each physical activity estimate was entered into a logistic model as a dichotomous variable (high/low activity) along with the following variables: sex, age, body mass index and waist-to thigh ratio

led for age, BMI and waist-to-thigh ratio, was significantly associated with 2-h post-load plasma glucose concentrations (Table 4). There was no association with fasting plasma glucose in males or females (data not shown).

\section{Historical physical activity and diabetes}

Individuals aged $37-59$ years $(n=353)$ were asked to estimate their former levels of physical activity as teenagers (12-18 years) and as younger (19-34 years) and older adults (35-49 years). As presented in Figure 3, the diabetic individuals reported considerably less historical and past year leisure physical activity than the non-diabetic subjects. In men, a similar finding was also noted for past year occupational physical activity. In contrast, most women did not report any occupational physical activity.

To examine further the relationship between leisure physical activity and non-insulin-dependent diabetes, reported leisure physical activity during each period of life (12-18 years, $19-34$ years and past year) was entered as a dichotomous variable (high/low; separated at the median
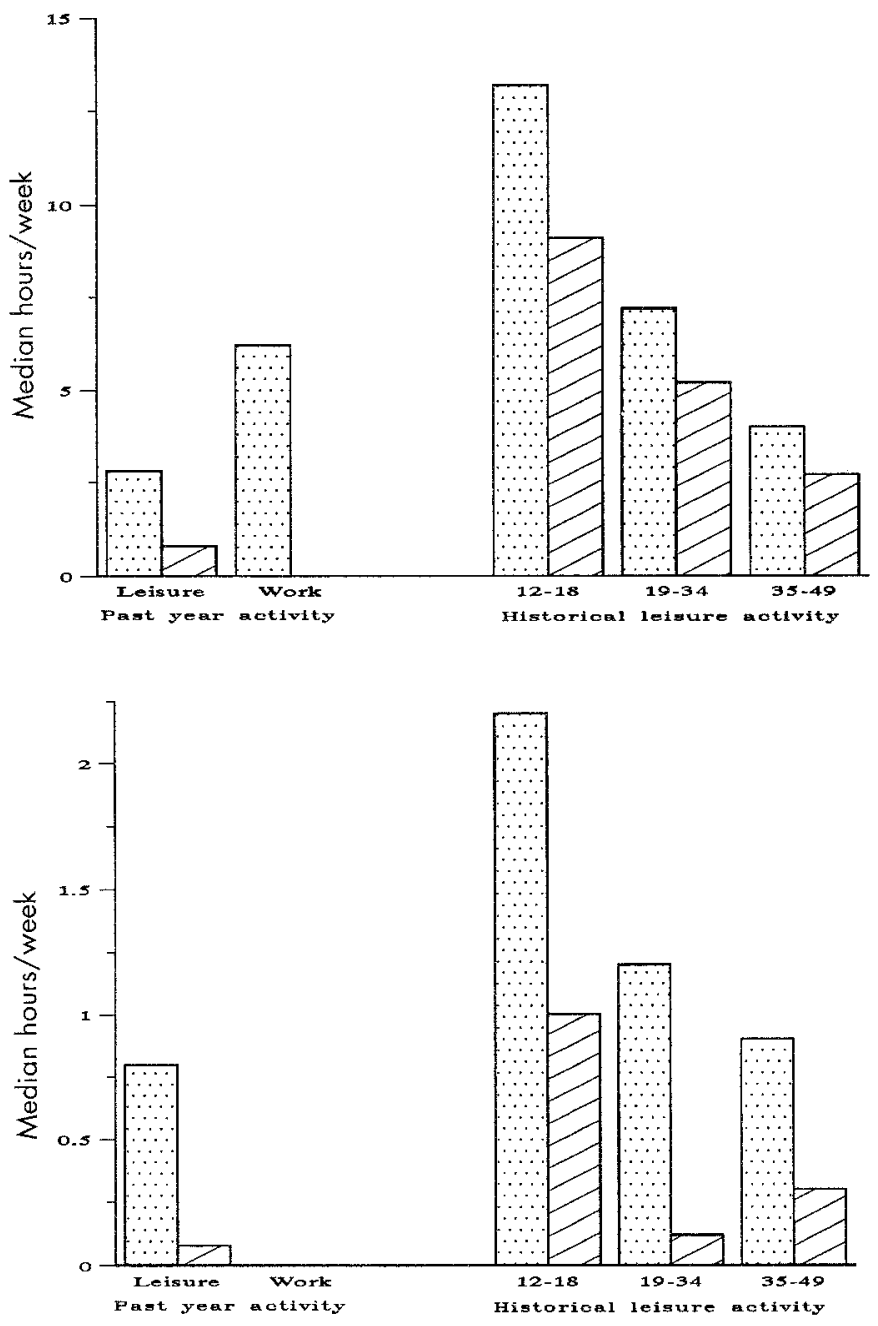

Fig. 3. Reported current leisure and occupational physical activity and historical leisure activity in diabetic and non-diabetic men (upper panel) and women (lower panel) aged 37-59 years, $\square$ with diabetes, $:$ without diabetes

for each sex) in a multiple logistic regression model with non-insulin-dependent diabetes as the dependent variable. As shown in Table 5, controlled for age, sex, BMI and waist-to-thigh ratio at the current examination (age 37-59 years), a high level of reported leisure physical activity as a teenager was inversely associated with non-insulin-dependent diabetes. Among individuals who reported more than the median amount of leisure physical activity as teenagers (12-18 years), the odds of having diabetes when examined at $37-59$ years of age was $45 \%$ of those reporting less activity. Estimated activity levels during the younger adult years (19-34 years) were also strongly inversely associated with diabetes at $37-59$ years of age (odds ratio $=0.47$ ). This was in contrast to the most recent time period in which the relationship between past year physical activity and diabetes prevalence was much weaker (odds ratio $=0.68$ ). In a model containing both historical and current leisure physical activity (not shown), the former remained significantly associated with diabetes. Treating past year physical activity as a continuous rather than categorical variable or adding current occupational physical activity to the leisure activity estimate 
strengthened the relationship between past year physical activity and non-insulin-dependent diabetes prevalence but did not alter the association between historical physical activity and diabetes prevalence.

\section{Discussion}

If physical activity influences the development of non-insulin-dependent diabetes, the following results would be expected when investigating the relationship between the two in a high-risk population such as the Pima Indians. First, an inverse relationship between current levels of physical activity and glucose intolerance would be anticipated. This relationship should remain even after excluding those with diagnosed diabetes in order to decrease the possibility that the physical inactivity is due to the diseased state. Secondly, when comparing subjects with and without non-insulin-dependent diabetes, those with diabetes would be expected to report less physical activity over their lifetime.

In this investigation, the prevalence of non-insulin-dependent diabetes was inversely related to the level of current physical activity. This relationship has been demonstrated in diverse ethnic groups [15] and could simply be the result of the disease state causing the inactivity. However, in the present study, a significant inverse relationship between levels of current physical activity and 2-h postload glucose concentrations was found in males, even after excluding those with diagnosed non-insulin-dependent diabetes. The lack of a similar relationship in females was most likely due to the extremely low levels of activity reported by females in this population.

The significant negative association between physical activity and post-load plasma glucose concentrations found in the Pima Indians is in agreement with findings from previous epidemiologic studies. Two-hour plasma glucose concentrations were significantly lower in active subjects of "both sexes in Asian Indians, Creole, and Chinese adults living in Mauritius [15]. In a study of nondiabetic Taiwanese men, plasma glucose concentrations were higher in office workers than in labourers doing heavy physical work [16]. In studies of non-diabetic participants in community health screening programmes, post-load glucose values were significantly lower in men who reported higher leisure physical activity levels (based upon questionnaire responses) than in those who reported little activity $[17,18]$. Another study of similar design found similar but non-significant trends in that the least active subjects tended to have higher glucose tolerance values [19]. In contrast, using a crude estimate of physical activity, the Whitehall study did not show any consistent relationship between physical activity levels and glucose tolerance [20].

The second anticipated result, less historical physical activity reported by diabetic than by non-diabetic individuals, was found in both sexes. When the contributions of historical and current physical activity were examined together, the former was more strongly associated with diabetes. This suggests that physical activity may have a protective role in the development of diabetes rather than re- duced physical activity just being the result of the disease. This hypothesis was previously suggested from three epidemiologic studies of college graduates and registered nurses. Women graduates who were former college athletes had a lower prevalence of diabetes than those who were non-athletes [21]. A more recent study of male graduates from the University of Pennsylvania [4] demonstrated that physical activity was inversely related to the incidence of non-insulin-dependent diabetes, a relationship that was particularly evident in men at high risk for developing diabetes (defined as those with a high BMI, a history of hypertension or a parentalhistory of diabetes). Finally, astudy of female registered nurses aged 34-59 years showed that women who reported engaging in vigorous exercise at least once a week developed non-insulin-dependent diabetes less often during the 8 years of follow-up than women who did not exercise weekly [5]. However, in all three of these prospective studies, the diagnosis of diabetes was based upon self-reported physician diagnosed diabetes.

Obesity and fat distribution are related to glucose intolerance in Pima Indians [13] as in other populations [15, $22]$. In the present study, physical activity was inversely associated with obesity and central distribution of fat (estimated by waist-to-thigh ratio), suggesting that the physical activity - glucose tolerance association might be due to an association between physical activity and these two anthropometric variables. However, obesity and fat distribution did not completely explain the relationship between physical activity and glucose tolerance in males. This suggests that this relationship is not mediated entirely through obesity and fat distribution but that physical activity has a protective role beyond that which can be accounted for by differences in obesity and fat patterning.

The fact that leisure but not occupational activity levels were more consistently related to plasma glucose concentrations, BMI, and waist-to-thigh ratio may reflect the general lack of occupational activity in the population. The distribution of leisure and occupational activities might also reflect a potential bias in this sample of the population since it is possible that those who volunteered for the study were more likely to be unemployed with more time to spend in leisure physical activities. Alternatively, the lack of association with occupational activity may be due to the fact that the occupational section of the activity questionnaire is less reproducible than the leisure activity section [8].

In summary, this cross-sectional study demonstrated that current physical activity was inversely associated with glucose intolerance, obesity and fat distribution, particularly in males. In addition, individuals with non-insulindependent diabetes reported less current and historical physical activity than non-diabetic subjects. Only a prospective study can determine the joint contributions of physical activity, obesity, and waist-to-thigh ratio to the development of diabetes. The continued collection of reported physical activity data in this population will eventually allow for such an evaluation.

Acknowledgements. We are indebted to the members of the Gila River Indian Community for participating in this investigation, to Ms. J.Jones, A. Barley, H. Johns and S. Antone for assisting in the 
data collection, and for the staff of the Diabetes and Arthritis Epidemiology Section for conducting the examinations. A.M.K. was supported in part by a National Institutes of Health cardiovascular training grant and later by a National Institutes of Health First Award grant. M.A.C. was supported by a postdoctoral fellowship award from the American Diabetes Association. R. G.N. was supported by a contract from the NIDDK.

\section{References}

1. Harris MI (1985) Prevalence of non-insulin-dependent diabetes and impaired glucose tolerance. In: Diabetes in America. U.S. Department of Health and Human Services. NIH Publication No. 85-1468, VI 1-31, Washington D.C.

2. Koivisto VA, Yki-Jarvinen H, DeFronzo RA (1986) Physical training and insulin sensitivity. Diab Metab Rev 1: 445-481

3. Despres JP, Tremblay A, Nadeau A, Bouchard C (1988) Physical training and changes in regional adipose tissue distribution. Acta Med Scand 723 [Supp1]: 205-212

4. Helmrich SP, Ragland DR, Leung RW, Paffenbarger RS (1991) Physical activity and reduced occurrence of non-insulin-dependent diabetes mellitus. N Engl J Med 325: 147-152

5. Manson JE, Rimm EB, Stampfer MJ et al. (1991) Physical activity and incidence of non-insulin-dependent diabetes mellitus in women. Lancet 338: 774-778

6. Bennett PH, Burch TA, Miller M (1971) Diabetes mellitus in American (Pima) Indians. Lasncet II: 125-128

7. Knowler WC, Bennett PH, Hamman RF, Miller M (1978) Diabetes incidence and prevalence in Pima Indians: a 19-fold greater incidence than in Rochester, Minnesota. Am J Epidemiol 108: 497-505

8. Kriska AM, Knowler WC, LaPorte RE et al. (1990) Development of questionnaire to examine relationship of physical activity and diabetes in Pima Indians. Diabetes Care 13:401-411

9. World Health Organization Expert Committee (1980) Second report on diabetes mellitus. Technical Report Series No. 646, Geneva

10. American College of Sports Medicine (1980) Guidelines for graded exercise testing and exercise prescription, 2 nd edn. Lea \& Febiger, Philadelphia

11. Kriska AM, Sandler RB, Cauley JA, LaPorte RE, Hom DL, Pambianco $G$ (1989) The assessment of historical physical activity and its relationship to adult bone parameters. Am J Epidemiol 127: $1053-1063$
12. U.S.Bureau of the Census (1984) Estimates of the population of the United States, by age, sex, and race: 1980 to 1983 . Current Population Reports, Series P-25, No. 949

13. Knowler WC, Pettitt DJ, Saad MF et al. (1991) Obesity in the Pima Indians: its magnitude and relationship with diabetes. Am J Clin Nutr 53: 1543 S-1551 S

14. Shimokata H, Tobin JD, Muller DC, Elahi D, Coon PJ, Andres R (1989) Studies in the distribution of body fat: I.Effects of age, sex and obesity. J Gerontol 44: M66-M73

15. Dowse GK, Zimmet PZ, Gareeboo H et al. (1991) Abdominal obesity and physical inactivity as risk factors for NIDDM and impaired glucose tolerance in Indian, Creole, and Chinese Mauritians. Diabetes Care 14:271-282

16. Wang JT, Ho LT, Tang KT, Wang LM, Chen YDI, Reaven GM (1989) Effect of habitual physical activity on age-related glucose intolerance. J Am Geriatr Soc 37: 203-209

17. Lindgärde F, Saltin B (1981) Daily physical activity, work capacity and glucose tolerance in lean and obese normoglycaemic middle-aged men. Diabetologia 20:134-138

18. Cederholm J, Wibell L (1985) Glucose tolerance and physical activity in a health survey of middle-aged subjects. Acta Med Scand 217:373-378

19. Montoye HJ, Block WD, Metzner H, Keller JB (1977) Habitual physical activity and glucose tolerance: males age 16-64 in a total community. Diabetes 26: 172-176

20. Jarrett RJ, Shipley MJ, Hunt R (1987) Physical activity, glucose tolerance, and diabetes mellitus: the Whitehall study. Diabetic Med 3: 549-551

21. Frisch RE, Wyshak G, Albright TE, Albright NL, Schiff I (1986) Lower prevalence of diabetes in female former college athletes compared with nonathletes. Diabetes 35: 1101-1105

22. Haffner SM, Stern MP, Hazuda HP, Rosenthal M, Knapp JA, Malina RM (1986) Role of obesity and fat distribution in non-insulin-dependent diabetes mellitus in Mexican Americans and non-Hispanic Whites. Diabetes Care 9: 153-161

Received: 29 December 1992

and in revised form: 27 April 1993

Dr. A. Kriska

Department of Epidemiology

Graduate School of Public Health

University of Pittsburgh

Pittsburgh, PA 15261

USA 